\title{
A Conceptual Framework for Intelligent Power Distribution Transformers
}

\author{
K. Somasena Reddy
}

\begin{abstract}
With the agenda of developing smart cities there is huge demand for continuous power supply. Power distribution transformers play avital role in providing a reliable power supply. Failure of a transformer will lead to interruptions in power supply. Many parameters lead to transformer failures. Health monitoring of transformer using IoT technology may help take proactive maintenance steps instead of reactive maintenance. When we combine IoT with AI it will more effective and IoT devices will take decision on their own. This paper presents a conceptual framework of this concept which makes the IoT devices in the transformers to make real-time decisions with the use of AI.
\end{abstract}

Keywords: Smart Cities, uninterrupted power supply, distribution transformer IoT, AI.

\section{INTRODUCTION}

Smart cities agenda is on the high note at the moment. India is soon to experience the lifestyle of smart cities. The Indian government has laid down an emphasis for development of 100 smart cities. Can one imagine smart cities without power? Of course, no. The country is demanding continuous power supply and to have an uninterrupted power supply [1]. In this mission of uninterrupted power supply for all, transformers have an important role to play by delivering reliable power supply to shape up the smart cities. Transformers play a key role in providing reliable power supply whether for infrastructure systems, industry or households. To keep these systems running, ensuring a sustainable power supply is of prime importance."

So, with rapid urbanization and industrialization, there is already high demand for uninterrupted power [2]. There is a possibility that this demand may double in the coming years due to smart cities initiative. To meet this demand India needs efficient power network, power distribution, supply systems etc., out which transformers will have the crucial role to play. Frequent failures of transformers will lead to interruptions in power supply and also generates big revenue loss to power distributors. Unfortunately, in India, the failure rate of these transformers is around $25 \%$ per annum. Proper maintenance, continuous monitoring, early \& prompt correction of any abnormal functioning of distribution transformers will increase its life and saves heavy investment, frequent replacements to the utilities and generates uninterrupted power supply. Therefore, real time monitoring of the Transformer is very necessary.

Important reasons for the failure of distribution

Revised Manuscript Received on March 18, 2020.

* Correspondence Author

K. Somasena Reddy*, Assistant Professor (Ad Hoc), Department of CSE, JNTUACEA, Ananthapuramu, India. E-mail somasena.cse@jntua.ac.in transformers includes vandalism or theft, low oil level in the transformer, overloading, unbalance loading, overheating of transformer oil, defective breather and consequent ingress of moisture [3]. The main causes of transformer oil overheating include insulation overheating, insulation liquid overheating, corona overheating, arcing overheating and screen-testing overheating [4]. This causes transformer failures and cause a huge problem in power distribution and also it unbalances the supply voltages. Once a transformer failed it can take a while to replace it.

Better the fitness of a transformer, longer the life. Issues in oil overheating referred to above are the principle reasons of decay of a transformer's fitness [5]. Earlier the health monitoring device was now not tons prevalent inside the distribution community and the faults could simplest be detected after a complete blackout. It induced predominant outcomes for the distribution machine as massive quantity of losses were incurred. Therefore, there was a foremost want for a reliable health monitoring system which would assist in creating preventive measures beforehand.

The present health tracking systems especially use IoT technology. IoT enables to invigilate the facts on-line of the key functional elements of the distribution transformers which offers constructive facts approximately the health of distribution-transformers which in flip will facilitate the services to apply their transformers in a great possible way and growth the existence of a transformer. IOT enables in identifying the troubles earlier i.e., before the occurrence of any failed mechanism which enables cost effective solutions and therefore much less penalty. IOT serves greater reliability and balance than other traditional systems [6].

Since IoT is only a network technology with a combination of sensors helps in sensing, and communicating data through network without human interaction and these IoT devices produce vast amount of data due to huge amount of IoT devices embedded in large number of transformers. This huge of amount data is to be transformed into valuable and actionable information within a given time period.

Obviously, it is not preferable to process this huge amount of data to take decisions at the server side (by the Central Power Control Centre) due to strict time constraints.

This is where Artificial Intelligence comes into play. Instead of transforming the sensed data to the control centre to further analysis and decision making, it will more effective if the IoT devices will take decision on their own. Therefore, when $\mathrm{AI}$ is added to the IoT [6-7], it means that those devices can analyse data and make decisions and act on the data without out involvement of humans or without transforming to the Central Control centre or to the cloud server. 


\section{A Conceptual Framework for Intelligent Power Distribution Transformers}

Thus, our proposed system is aimed in developing a framework for Intelligent Power Distribution Transformers which drive efficiency and effectiveness. The combo of AI and IoT devices makes the IoT devices in the transformer to analyze data locally to predict the transformer failures and then uses this information to make moment-to-moment decisions about how to respond to the oil overheating.

\section{LITERATURE SURVEY}

There are various existing mechanism for power distribution transformer health monitoring. All of them can be classified into two categories.

\section{A. Manual Monitoring}

In manual monitoring mechanism a person visits the transformer site at regular intervals to record parameters of importance. But this method of monitoring distribution transformers doesn't represent information about oil levels, and vandalism or theft can be identified after it has happened.

\section{B. IoT based Mechanisms}

Health monitoring of distribution transformers using IOT [8-21] assist to update the equipment earlier than failure and continuity of the electricity will no longer be disturbed. The IoT based health tracking system works in actual time and makes use of temperature-sensor, capacity transformer and contemporary transformer for monitoring temperature, voltage and modern of the distribution transformer and ship these information to a far off server where it can be monitored and vital action can be taken to keep away from the outage of the electricity supply.

\section{PTOPOSED SYSTEM}

As we mentioned earlier, as the volume of data form IoT devices keeps increasing, consequently, we need efficient mechanisms to quickly analyse and make decisions. Hence it is more preferable to have IoT enabled AI solutions for power distribution transformers to predict when it works improperly and to fix the transformers and power supply before they break, which save from disasters that will occur.

Figure 1 illustrates a conceptual diagram of the proposed approach. Figure 2 illustrates the flow of activities in the proposed approach. Figure 3 illustrates a conceptual algorithm for the proposed approach.

The AI enabled IoT sensor contain an intelligence component and take steps to reduce the load of a transformer step by step before it fails or a service provider reaches to the location and rectify the problem of transformer.

For example, consider a transformer that supply power to industries, hospitals, villages, schools etc. Due to transformer oil heating or any other problem the transformer will get over heated or overloaded. Then the embedded Intelligence Component in the transformer takes steps to cutoff power supply moment-by-moment or entirely disables the transformer by applying the following algorithm.

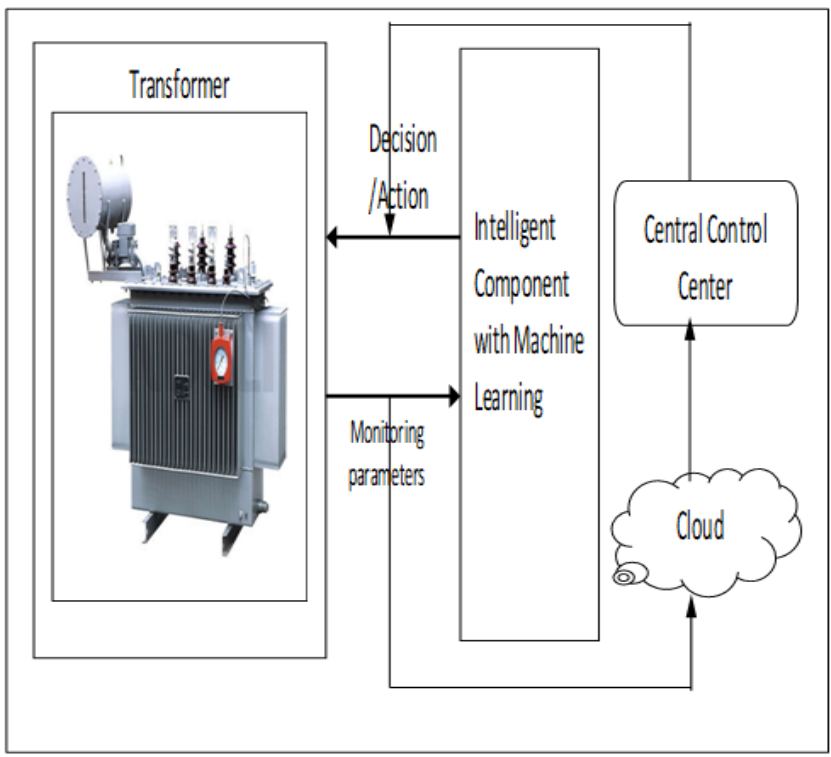

Fig. 1.IoT and AI Enabled Distribution Transformer

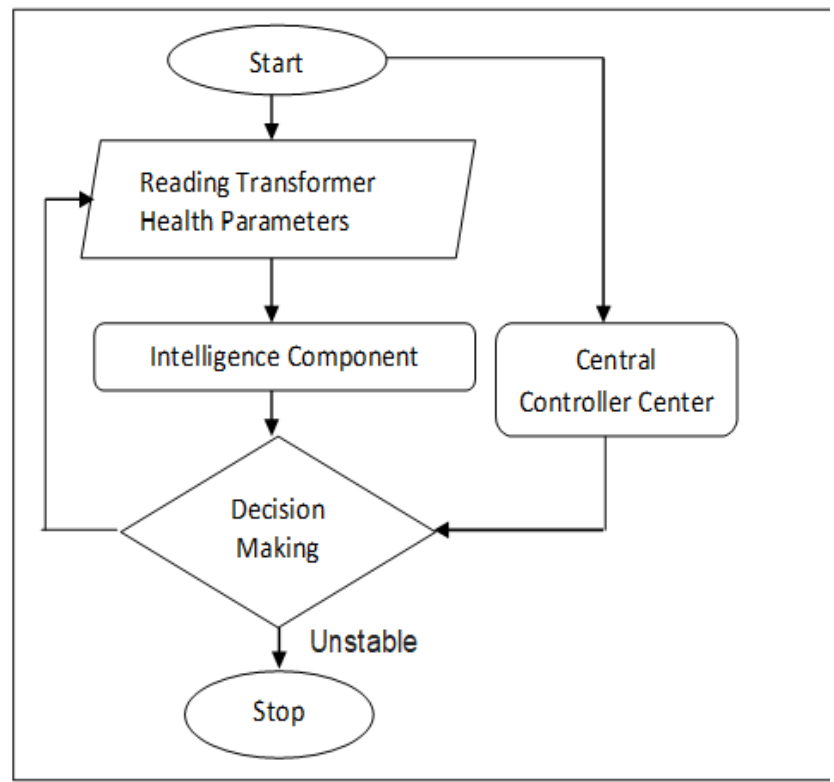

Fig. 2. Flowchart of the Proposed Approach.

By applying these AI enabled IoT temperature/overload of transformer can be reduce without any interference of controller/service provider.

\section{CONCLUSION}

In this paper a conceptual framework for intelligent power distribution transformers is proposed. With a rapid urbanization and industrialization, there is a high demand for uninterrupted power supply. Frequent failures of transformers will lead to interruptions in power supply and also generates big revenue loss to power distributors. The present transformers' health monitoring systems mainly use IoT technology. 


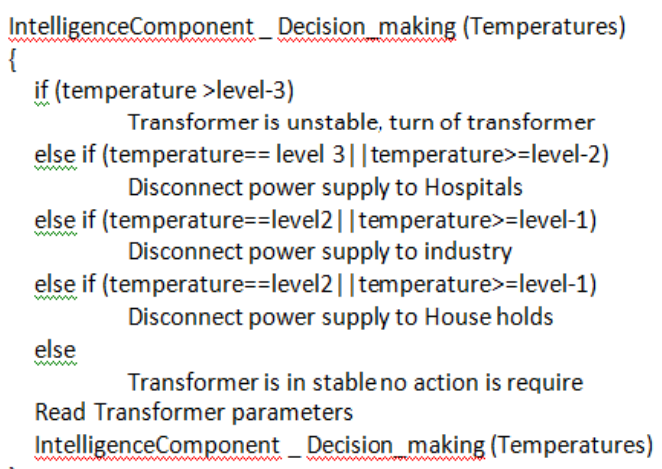

Fig. 3. Pseudocode for the proposed approach.

When we combine IoT with AI it will more effective and IoT devices will take decision on their own. The combo of AI and IoT devices makes the IoT devices in the transformer to analyse data locally, predicts the malfunctioning of transformer and fix the transformers and power supply before they break, which save from disasters that will occur. Since this proposal is only a conceptual one, implementation of this approach in a real environment is left for feature.

\section{REFERENCES}

1. R. Ramakrishnan and L. Gaur, "Smart electricity distribution in residential areas: Internet of Things (IoT) based advanced metering infrastructure and cloud analytics," 2016 International Conference on Internet of Things and Applications (IOTA), Pune, 2016, pp. 46-51.

2. R. Garai, P. Maity, R. Hossain, P. Roy and T. K. Rana, "Smart village," 2017 1st International Conference on Electronics, Materials Engineering and Nano-Technology (IEMENTech), Kolkata, 2017, pp. 1-6.

3. Abu Jahal, C., "Causes of transformer failures and diagnostic methods - A review," Renewable and Sustainable Energy Reviews (2017), Volume 82, Part 1, February 2018, Pages 1442-1456.

4. D. S., Prathibha Suresh, T., Kouser Taj, "Oil Based Transformer Health Monitoring System", International Journal of Science and Research (IJSR) ISSN (Online): 2319-7064 Impact Factor, vol. 3.358, 2012.

5. K. Ibrahim, R. M. Sharkawy, H. K. Temraz and M. M. A. Salama, "Selection criteria for oil transformer measurements to calculate the Health Index," in IEEE Transactions on Dielectrics and Electrical Insulation, vol. 23, no. 6, pp. 3397-3404, Dec. 2016.

6. W. Peng, W. Gao and J. Liu, "AI-Enabled Massive Devices Multiple Access for Smart City," in IEEE Internet of Things Journal, vol. 6, no. 5, pp. 7623-7634, Oct. 2019.

7. "Towards intelligent IoT smart city platform based on OneM2M guideline: Smart grid case study", Proc. IEEE Int. Conf. Big Data Smart Comput. (BigComp), pp. 701-704, Jan. 2018.

8. Xiao-Hui Cheng, Yang Wang, "The remote monitoring system of transformer fault based on The internet of Things", 2011 International Conference on Computer Science and Network Technology.

9. A. Kumar, A. Raj, A. Kumar, S. Prasad, B. Kumar, "Method for monitoring of distribution transformer", Undergraduate Academic Research Journal (UARJ), 2012.

10. Rakesh Kumar Pandey, Dilip Kumar. "Distributed Transformer Monitoring System Based On Zigbee Technology". International Journal of Engineering Trends and Technology (IJETT). V4(5):1981-1983 May 2013.

11. Monika Agarwal, Akshaypandya, "GSM Based Condition Monitoring of Transformer", IJSRD - International Journal for Scientific Research \& Development, vol. 1, no. 12, 2014.

12. P.M. Sneha Angeline, "Performance Monitoring of Transformer Parameters, International Journal of Innovative Research in Electrical, Electronics, Instrumentation and Control Engineering, vol. 3, no. 8, August 2015, pp. 49-51.

13. U.V. Patil, Kathe Mohan, Harkal Saurabh, Warhade Nilesh, "Transformer Health Condition Monitoring Using GSM Technology", IJARIIE, vol. 2, no. 2, 2016, ISSN (O)-2395-4396.
14. R. R. Pawar and S. B. Deosarkar, "Health condition monitoring system for distribution transformer using Internet of Things (IoT)," 2017 International Conference on Computing Methodologies and Communication (ICCMC), Erode, 2017, pp. 117-122.

15. R. R. Pawar, P. A. Wagh and S. B. Deosarkar, "Distribution transformer monitoring system using Internet of Things (IoT)," 2017 International Conference on Computational Intelligence in Data Science (ICCIDS), Chennai, 2017, pp. 1-4

16. T. A. Kumar and A. Ajitha, "Development of IOT based solution for monitoring and controlling of distribution transformers," 2017 International Conference on Intelligent Computing, Instrumentation and Control Technologies (ICICICT), Kannur, 2017, pp. 1457-1461.

17. D. Srivastava and M. M. Tripathi, "Transformer Health Monitoring System Using Internet of Things," 2018 2nd IEEE International Conference on Power Electronics, Intelligent Control and Energy Systems (ICPEICES), Delhi, India, 2018, pp. 903-908.

18. N. Mussin et al., "Transformer Active Part Fault Assessment Using Internet of Things," 2018 International Conference on Computing and Network Communications (CoCoNet), Astana, 2018, pp. 1-6.

19. M. Bagheri, S. Nezhivenko, M. Salay Naderi, A. Zollanvari, "A new vibration analysis approach for transformer fault prognosis over cloud environment", International Journal of Electrical Power \& Energy Systems, vol. 100, pp. 104-116, 2018.

20. T. K. Roy and T. K. Roy, "Implementation of IoT: Smart Maintenance for Distribution Transformer using MQTT," 2018 International Conference on Computer, Communication, Chemical, Material and Electronic Engineering (IC4ME2), Rajshahi, 2018, pp. 1-4.

21. W. K. A. Hasan, A. Alraddad, A. Ashour, Y. Ran, M. A. Alkelsh and R. A. M. Ajele, "Design and Implementation Smart Transformer based on IoT," 2019 International Conference on Computing, Electronics \& Communications Engineering (iCCECE), London, United Kingdom, 2019, pp. 16-21.

\section{AUTHORS PROFILE}

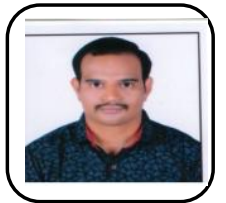

Mr. K Somasena Reddy is currently working as an Assistant Professor (Ad Hoc) at Department of CSE, JNTUACEA, Ananthapuramu. He obtained his M. Tech. degree in Computer Science from JNTUCEA, JNTUA, Ananthapuram. His research interests include, Internet of Things, Software Defined Networks, Artificial Intelligence, and Machine 